\title{
Radiographic and Clinical Factors in Pediatric Patients with Surgical Small-Bowel Intussusception
}

AUTHORS: Robert J. Vandewalle MD MBA ${ }^{1}$, Alexis K. Bagwell MD MS ${ }^{1}$, Jared R. Shields $\mathrm{MD}^{2}$, R. Cartland Burns $\mathrm{MD}^{1}$, Brandon P. Brown MD MA², Matthew P. Landman MD MPH ${ }^{1}$

AFFILIATION:

${ }^{1}$ Indiana University School of Medicine, Department of General Surgery, Division of Pediatric Surgery, Indianapolis, Indiana

${ }^{2}$ Indiana University School of Medicine, Department of Radiology, Division of Pediatric Radiology, Indianapolis, Indiana

CORRESPONDING AUTHOR:

Name: Matthew P. Landman, MD, MPH

Address: 705 Riley Hospital Dr \#2500; Indianapolis, IN 46202

Telephone: 317-274-4681

Fax: 317-274-4491

Current E-mail: landman@iu.edu

DISCLAIMERS: All authors have no conflicts of interest to disclose.

FUNDING: There is no funding for this paper.

AUTHOR CONTRIBUTION:

Study Conception and Design: Robert Vandewalle, R. Cartland Burns, Matthew Landman Data Acquisition: Robert Vandewalle, Alexis Bagwell, Jared Shields, Joseph Easton, Brandon Brown Analysis and Data Interpretation: Robert Vandewalle, R. Cartland Burns, Brandon Brown, Matthew Landman

Drafting of the Manuscript: Robert Vandewalle, Alexis Bagwell, Matthew Landman Critical Revision: Robert Vandewalle, R. Cartland Burns, Brandon Brown, Matthew Landman

LEVEL OF EVIDENCE: Level II

This is the author's manuscript of the article published in final edited form as:

Vandewalle, R. J., Bagwell, A. K., Shields, J. R., Burns, R. C., Brown, B. P., \& Landman, M. P. (2019). Radiographic and clinical factors in pediatric patients with surgical small-bowel intussusception. Journal of Surgical Research, 233, 167-172. https://doi.org/10.1016/j.jss.2018.08.002 


\section{Abstract:}

Background: When evaluating a pediatric patient with abdominal pain, identification of a small-bowel to small-bowel intussusception (SBI) on radiologic imaging can create a diagnostic dilemma. The clinical significance and need for surgical exploration of SBI is highly variable, as most of them are considered clinically insignificant. We hypothesize that combination of clinical and radiologic factors in an exclusively SBI population will yield factors that guide the clinician in making operative decisions. Methods: A comprehensive database from a pediatric tertiary hospital was reviewed from 1/1/2011 to $12 / 31 / 2016$ for any radiographic study mentioning intussusception. Results were reviewed for patients having only SBI (i.e. not ileo-colic intussusception) and this comprised the study cohort. The electronic medical records for these patients were reviewed for clinical presentation variables, need for operative intervention, and identification of the intussusception during surgery. Patients with SBI due to enteral feeding tubes were excluded from the study.

Results: Within the study period, 139 patients were identified with a small-bowel intussusception (SBI) on radiologic imaging. Univariate analysis yielded numerous clinical and radiologic factors highly predictive for need for surgical intervention. However upon multivariate analysis, only a history of prior abdominal surgery (OR: 7.2; Cl: 1.1-46.3), the presence of focal abdominal pain (OR: $22.1 ; 4.2-116.3$ ), and the intussusception length (cm; OR: 10.6; $\mathrm{Cl}$ : 10.3-10.8) were correlated with need for surgical intervention.

Conclusions: Small-bowel to small-bowel intussusception is a disease process with a highly variable clinical significance. The presence of focal abdominal pain, a history of prior abdominal surgery, and the intussusception length are the greatest predictors for need for operative intervention. 


\section{Introduction:}

When evaluating a pediatric patient with abdominal pain, identification of a small-bowel to small-bowel intussusception (SBI) on radiologic imaging can create a diagnostic dilemma. Most SBI are an unexpected finding on radiographic imaging, and many clinicians regard the majorities of these as transient/clinically insignificant. However, the reported clinical significance and need for surgical exploration of SBI is highly variable across the literature. ${ }^{1-3}$ Indeed, even in patients with disease conditions known to cause "pathologic lead points", surgical intervention may not be necessary. ${ }^{3-6}$ Conversely, Ko et. al reported a high rate of SBI requiring surgical intervention with a high associated delay to diagnosis and complication rate (i.e. ischemic bowel/perforation). ${ }^{2}$ Therefore a consulted surgeon must recognize when, although likely rare, SBI are in fact require surgical intervention.

Several single institution reports have noted radiologic factors predicting need for surgical intervention in the setting of SBI, and similarly others have noted clinical factors that may favor operative intervention in this patient population. ${ }^{6-13}$ However, at this time combining both clinical and radiologic factors have only been described in a mixed population of patients with both SBI and ileocecal/ileo-colic/colo-colic intussusception. ${ }^{14}$ We hypothesize that combination of clinical and radiologic factors in an exclusively SBI population will yield factors that guide the clinician in making operative decisions.

\section{Methods:}

The digital radiologic database at IU Health, including Riley Hospital for Children, is searchable based upon factors including keywords in the study report, imaging modality, age of patient, and indication for imaging. Within this database, a search for all patients with the mention of "intussusception" within the radiology report was completed. Resultant studies were filtered for only abdominal sonographic studies (US) and abdominal computed tomography (CT) in patients evaluated at Riley Hospital for Children under the age of 18 years from $1 / 1 / 2011$ to $12 / 31 / 2016$. This set of studies 
were then reviewed for details of the type of intussusception within the radiology report. Those studies with report of ileocolic intussusception were excluded. Patients with SBI due to enteral feeding tubes were also excluded. For patients with multiple radiologic studies completed at the same hospitalization, only the initial imaging study identifying the SBI was included in analysis. This cohort represented all patients with small-bowel to small-bowel intussusception (SBI). Institutional Review Board approval was obtained for this study.

The identified studies were then reviewed by radiology staff/residents (B.B. and J.S.) and confirmed by radiology staff (B.B.) within Riley Hospital for Children's Radiology Department for multiple factors thought to contribute to the need for surgical intervention in children with SBI. Reviewers were blinded to patient outcome at the time of radiologic review. Multiple radiologic factors were reviewed (Table 2). Six patients were identified that did not have adequate radiologic studies for review (four underwent US; two CT) but their clinical factors were included in the analysis.

Concurrently, multiple clinical factors related to those patients identified from the radiologic database were reviewed (Table 3). These factors included the presence of abdominal pain, duration of abdominal pain, localized versus general abdominal pain, presence/duration of emesis/constipation/obstipation, history of prior abdominal surgery, and history of condition predisposing to SBI. Due to the retrospective nature of the study not all clinical and radiographic variables were available for review, therefore the number of patients with reportable data points is given for each clinical variable where all were not retrievable. Patients were deemed to have a SBI that required surgical treatment (SSBI) if an intussusception was identified at the time of surgery and reported within the corresponding operative note. Those patients who underwent non-therapeutic exploratory laparoscopy/laparotomy (i.e. no intussusception at the time of surgery) were deemed to not have an SSBI. Univariate statistical analysis was completely using Chi-square analysis and two-sample t tests where appropriate. All factors with $p<0.1$ were then compiled and underwent logistic regression 
analysis with the primary outcome variable being SSBI in all analyses. Statistical significance was set at 0.05 or less.

\section{Results:}

\section{Demographics}

Within the study period, 139 patients were identified with a small-bowel intussusception (SBI) on radiologic imaging (see table 1). All patients had a complaint of abdominal pain at the time of imaging. The average age was 5.3 years old and 56 patients were female (56/139; 40.3\%). Six patients had a history of either a purpuric or polyposis syndrome $(6 / 139 ; 4.3 \%)$. Within the study cohort, 18 patients underwent surgery $(18 / 139 ; 12.9 \%)$ and 11 were found to have an intussusception at the time of operation (11/139; 7.9\%). Seventy-one (71/139; 51.0\%) patients underwent $\mathrm{CT}$ as the initial imaging modality, and 69 (69/139; 49.0\%) had US completed initially. There were no significant differences among patients with and without SSBI based upon demographic variables (i.e. age and gender). Among the 11 patients who had SSBI, 4 had identified polyps at their SBI, 3 patients had lymphoid hyperplasia, 2 patients had Henoch-Schonlein purpura, and 2 had a history of prior abdominal surgery (1 Wilms tumor resection; 1 ileocolic intussusception reduction). Of note, 1 patient with Juvenile Polyposis Syndrome and 1 patient with Henoch-Schonlein purpura were diagnosed only after their presentation with SSBI. Both patients with a history of prior abdominal surgery underwent manual reduction and the remainder underwent segmental small-bowel resection and/or polypectomy. TABLE 1

\section{Univariate Analysis}

Radiographic variables

Chi-squared analysis of categorical radiologic variables found statistically significant correlations between free fluid, fat stranding and bowel dilation proximal to the intussusception and SSBI (table 2). There were no significant associations with hyperemia $(p=0.736)$, location of the intussusception 
(0.346), the presence of lymphadenopathy $(p=0.150)$ and presence of peristalsis within in the intussusception $(p=0.791)$.

Evaluation of continuous radiologic variables found statistically significant differences in intussusception length ( $9.0 \mathrm{vs} .2 .7 \mathrm{~cm})$, and bowel wall thickness $(7.7 \mathrm{vs} .4 .3 \mathrm{~mm})$ for patients with and without SSBI, respectively. Outer SBI diameter was not significant ( $p=0.12)$. TABLE 2.

\section{Clinical variables}

Chi-squared analysis was completed on binary clinical outcome variables (table 3). All patients presented with complaints of abdominal pain, therefore this was not examined as a binary independent variable. Significant correlations between a history of prior abdominal surgery, presence of constipation/obstipation and endorsement of focal pain at the time of presentation and SSBI were noted. There were no significant associations with diffuse abdominal pain $(p=0.504)$, presence of peritonitis (on, $p=0.497)$, any history of associated emesis $(p=0.18)$, and known prior polyp or purpuric syndrome $(p=0.135)$.

Continuous clinical variables were then analyzed (table 3). There was a significant difference in duration of constipation (1.0 vs. 0.3 days) for patients with and without SSBI, respectively. Duration of pain (14.1 vs. 5.2 days) approached but did not reach significance $(p=0.051)$. There were no significant associations with duration of nausea $(p=0.465)$, duration of emesis $(p=0.994)$, and duration of obstipation ( $p=0.872)$. TABLE 3

\section{Multivariate Regression Analysis}

Multivariate logistic regression analysis was then completed using all variables found on univariate analysis to have $p \leq 0.10$ (see table 4). Due to a high level of collinearity with intussusception length, bowel wall thickness was removed from the model during regression. With respect to clinical and radiologic factors, regression analysis excluded all factors other than length of intussusception, history of prior abdominal surgery and presence of focal abdominal pain. TABLE 4. 


\section{Discussion:}

A delicate balance must be achieved when evaluating a pediatric patient with SBI. While the vast majority of these patients will not require operative intervention, a small percentage will. For this small percentage, delays in diagnosis can have catastrophic consequences. Therefore, the clinician must balance over treatment of the many with under treatment of the few. It was with these concerns in mind this study was crafted. We identified all patients with radiologic evidence of a small-bowel to small-bowel intussusception and identified radiologic and clinical factors that were associated with the need for surgical treatment. During the study period 139 patients met study criteria, of which 11 required operative intervention. The number of patients and homogeneity of the diagnosis (i.e. only small-bowel to smallbowel intussusception) makes this study unique strong for analysis. Review of radiologic and clinical data for these patients revealed multiple factors that based upon univariate analysis were highly predictive for need for surgical intervention (tables $2 \& 3$ ). However upon multivariate analysis, only a history of prior abdominal surgery, the presence of focal abdominal pain, and the intussusception length were predictive (table 4).

Although identification and radiologic evaluation of SBI using both US and CT modalities has been well described, the current literature is mixed regarding the need for operative intervention for SBI. ${ }^{6,8,15,16}$ While Kornecki et al. and Doi et al. reported that a large number of incidentally-found SBI will spontaneously reduce, Ko et al., Koh et al., and others have reported that the need for intervention is significantly high. ${ }^{1-4}$ Within our study cohort of exclusively SBI, $7.9 \%$ of patients required an operation for resolution of their intussusception. All patients underwent surgery for persistent abdominal pain with 5 of the 11 patients with complaints of emesis. No patients developed peritonitis within our cohort, and there were no additional symptoms reported within the medical records for justification for surgery. This is in contrast to Ko et al. where vomiting (89.5\%), abdominal pain and/or irritable crying 
(89.5\%), fever (52.6\%), bloody stools (26.3\%), palpable abdominal masses (15.8\%), hematemesis (10.5\%), jaundice (5.3\%), and seizures (5.3\%) were present at the time of surgery. ${ }^{2}$

Additionally, there is significant variance within the literature regarding the presence of "pathologic" lead points (i.e. polyps, purpuric lesions, diverticulum, etc) but not lymphoid hyperplasia as a cause for SBI. Munden et al. and Doi et al. reported that none of the patients in their study who had SSBI had an identified pathologic lead point. ${ }^{4,10}$ Conversely, Koh et al. reported that 5 out of 6 of their patients with SSBI had an identifiable pathologic lead point. Within our study population, 6 of 11 patients with SSBI had a pathologic lead point. It is important to note that only 4 of these 6 patients carried a diagnosis concerning for formation of lead points prior to their presentation with SSBI.

Multivariate analysis revealed two clinical factors highly predictive of SSBI within our study cohort: presence of focal abdominal pain and a history of prior abdominal surgery. To our knowledge, the presence of focal abdominal pain has never been identified as a risk factor for SSBI. Conversely, SSBI following abdominal surgery is widely reported. ${ }^{9,13,17}$ In addition, the development of SSBI specifically after surgery for abdominal malignancy is also well reported. ${ }^{7,12}$ In this study cohort, the mean time to develop a SBI was 1235 days and the earliest SSBI was 2115 days after surgery. Within our study population, four patients with SSBI had a history of prior abdominal surgery, one of which had a history of Wilms tumor. While focal abdominal pain could be considered nebulous, we feel this result, in the proper setting, should prompt, at a minimum close observation. Similarly, duration of abdominal pain in our cohort was quite variable, and at times difficult to determine acute versus chronic abdominal pain. In these setting the full duration of reported abdominal pain was used. Ten patients reported abdominal pain $>30$ days. One of these patients who complained of abdominal pain for 120 days had an SSBI, the remainder did not. Sub-group analysis was completed excluding these 10 patients, and once again the difference in pain approached but did not reach significance between SSBI and no SSBI. 
Numerous reports have cited radiologic factors influencing the need for surgery in the setting of SBI. In a mixed group of SBI and intussusception involving the large bowel, Zhang et al. reported differences in intussusception length $(6.0$ vs. $5.5 \mathrm{~cm})$ and diameter $(3.6 \mathrm{vs} .3 .1 \mathrm{~cm})$ for those patients requiring surgery and those that did not. ${ }^{14}$ Clinical variables of intussusception location, presence of obstipation/constipation, bowel dilation outside the intussusception, and presence of free fluid were also reported as significant. ${ }^{14}$ Neither multivariate analysis nor receiver operator characteristic curves were reported, as the primary goal of this study was to determine if pathologic lead points could be identified on ultrasound. Munden et al. reported that that among 35 patients with SBI (of which 13 were SSBI), all non-SSBI had a diameter $<3.5 \mathrm{~cm}$ and all but one SSBI was $>3.5 \mathrm{~cm}{ }^{10}$. Utilizing $3.5 \mathrm{~cm}$ as a cut off for operative intervention yielded a sensitivity and specificity of $93 \%$ and $100 \%$, respectively ${ }^{10}$. Ko et al. reported that among $19 \mathrm{SBI}$ the average length was $2.9 \mathrm{~cm}$, and the average bowel wall thickness was $7.2 \mathrm{~mm} .{ }^{2}$ Zhang et al. reported that among 56 patients with SBI/ileocolic intussusception, 17 patients underwent surgery. ${ }^{13}$ Among these 17 patients, two did not have an intussusception at the time of surgery but were included in the statistical analysis. Analysis of sonographic variables found a significant difference in intussusception length $(6.5 \mathrm{vs} .3 .0 \mathrm{~cm})$, intussusception diameter $(2.8 \mathrm{vs} .1 .8 \mathrm{~cm})$, intussusception outer rim thickness $(0.55$ vs. $0.35 \mathrm{~mm})$, location of intussusception, presence of free fluid, and bowel wall dilation outside of the intussusception. ${ }^{13}$ Multivariate analysis found significance for all three intussusception measurements, and cut-off values based upon receiver operating characteristic curves were reported. Within the current study, which was based solely upon evaluating $\mathrm{SBI}$, multivariate analysis found a high level of collinearity between intussusception length/diameter/wall thickness. However, the high odds ratio for SBI length within our study is consistent with these previously reported findings.

Our study is not without notable limitations. Due to the retrospective nature, several clinical and radiologic data points were not obtainable. These deficiencies, along with the inherent nature of a 
retrospective review, limits the strength of our conclusions and may confer unrecognized biases in the collected data. While the study cohort consisted of 139 patients, only 11 had SSBI, which limits the strength of the identified radiographic/clinical variables. A study designed to overcome this factor would require a multi-institutional collaboration and/or a prolonged accrual period. Additionally, there are likely differences in the sensitivities of CT and US for detecting SBI. This may serve as another limitation to this study. However, the objective of this study was to determine which SBI, when found incidentally on imaging for abdominal pain, should be approached with surgical correction. The smallest SSBI identified in our study was $2.3 \mathrm{~cm}$ in length. While not conclusive, this would suggest either modality should be adequate to identify SSBI. To our knowledge, there are no reports of the comparative efficacy of detecting SSBI using CT versus US. Recognizing these limitations, to our knowledge this the largest study focusing solely upon small-bowel to small-bowel intussusception and the factors that may suggest the need for operative intervention. Two prior recognized factors (i.e. prior abdominal surgery and length of intussusception) were corroborated within our study and an additional clinical factor (i.e. presence of focal abdominal pain) were identified on multivariate regression analysis.

\section{Conclusion:}

Small_bowel to small_bowel intussusception is a disease process with a highly variable clinical significance. While the vast majority of patients will not require anything more than observation, a small subset of patients will require surgery. Delaying operative intervention for this subset has the potential to increase the risk of morbidity and mortality. Based upon this single institution review, the presence of focal abdominal pain, a history of prior abdominal surgery, and the intussusception length are the greatest predictors for need for operative intervention. Due to the low incidence of surgical intervention for SSBI, a larger or even multi-institutional review is needed to corroborate these findings.

Acknowledgment: We would like to thank Lava Timsina, M.P.H., Ph.D. at the Center for Outcomes Research Education in Surgery (C.O.R.E.S.) within Indiana University School of Medicine Department of Surgery for his assistance in statistical analysis for this manuscript 


\section{$\underline{\text { References }}$}

1. Kornecki A, Daneman A, Navarro O, Connolly B, Manson D, Alton DJ. Spontaneous reduction of intussusception: clinical spectrum, management and outcome. Pediatr Radiol. 2000;30(1):58-63.

2. Ko SF, Lee TY, Ng SH, et al. Small bowel intussusception in symptomatic pediatric patients: experiences with 19 surgically proven cases. World J Surg. 2002;26(4):438-443.

3. Koh EP, Chua JH, Chui $\mathrm{CH}$, Jacobsen AS. A report of 6 children with small bowel intussusception that required surgical intervention. J Pediatr Surg. 2006;41(4):817-820.

4. Doi O, Aoyama K, Hutson JM. Twenty-one cases of small bowel intussusception: the pathophysiology of idiopathic intussusception and the concept of benign small bowel intussusception. Pediatr Surg Int. 2004;20(2):140-143.

5. Siaplaouras J, Moritz JD, Gortner L, Alzen G. [Small bowel intussusception in childhood]. Klin Padiatr. 2003;215(2):53-56.

6. Strouse PJ, DiPietro MA, Saez F. Transient small-bowel intussusception in children on CT. Pediatr Radiol. 2003;33(5):316-320.

7. Cohen MD, Baker M, Grosfeld JL, Siddiqui A. Post-operative intussusception in children with neuroblastoma. Br J Radiol. 1982;55(651):197-200.

8. Ko SF, Tiao MM, Hsieh CS, et al. Pediatric small bowel intussusception disease: feasibility of screening for surgery with early computed tomographic evaluation. Surgery. 2010;147(4):521528.

9. Mollitt DL, Ballantine TV, Grosfeld JL. Postoperative intussusception in infancy and childhood: analysis of 119 cases. Surgery. 1979;86(3):402-408. 
10. Munden MM, Bruzzi JF, Coley BD, Munden RF. Sonography of pediatric small-bowel intussusception: differentiating surgical from nonsurgical cases. AJR Am J Roentgenol. 2007;188(1):275-279.

11. Rajagopal R, Mishra N, Yadav N, Jhanwar V, Thakur A, Mannan N. Transient versus surgically managed small bowel intussusception in children: Role of ultrasound. Afr J Paediatr Surg. 2015;12(2):140-142.

12. VanHouwelingen LT, Seims AD, Ortega-Laureano L, et al. Use of ultrasound in diagnosing postoperative small-bowel intussusception in pediatric surgical oncology patients: a singlecenter retrospective review. Pediatr Radiol. 2018;48(2):204-209.

13. Zhang Y, Bai YZ, Li SX, Liu SJ, Ren WD, Zheng LQ. Sonographic findings predictive of the need for surgical management in pediatric patients with small bowel intussusceptions. Langenbecks Arch Surg. 2011;396(7):1035-1040.

14. Zhang Y, Dong Q, Li SX, et al. Clinical and Ultrasonographic Features of Secondary Intussusception in Children. Eur Radiol. 2016;26(12):4329-4338.

15. Park NH, Park SI, Park CS, et al. Ultrasonographic findings of small bowel intussusception, focusing on differentiation from ileocolic intussusception. Br J Radiol. 2007;80(958):798-802.

16. Tiao MM, Wan YL, Ng SH, et al. Sonographic features of small-bowel intussusception in pediatric patients. Acad Emerg Med. 2001;8(4):368-373.

17. Guney LH, Fakioglu E, Acer T, et al. Is every intussusception treatment an emergency intervention or surgery? Ulus Travma Acil Cerrahi Derg. 2016;22(2):139-144. 
Radiographic Factors

\begin{tabular}{|l|l|}
\hline Outer diameter of SBI $(\mathrm{mm})$ & Intraabdominal free fluid (yes/no) \\
\hline Greatest bowel wall thickness $(\mathrm{mm})$ & Fat-stranding (yes/no) \\
\hline Length of $\mathrm{SBI}(\mathrm{cm})$ & Hyperemia (yes/no) \\
\hline $\begin{array}{l}\text { Location of SBI } \\
\text { (LUQ/LLQ/RUQ/RLQ/midabdomen) }\end{array}$ & Lymphadenopathy (yes/no) \\
\hline Bowel dilation outside intussusception & Peristalsis visible in intussusception (on US only) \\
\hline
\end{tabular}

Table 1. Radiographic Factors Analyzed on Ultrasound (US) of Computed Tomography (CT) studies

Clinical Factors

\begin{tabular}{|l|l|}
\hline Known Purpuric or Polyposis Syndrome (yes/no) & Duration of pain (1/2 day intervals) \\
\hline Focal Pain (yes/no) & Duration of nausea ( $1 / 2$ day intervals \\
\hline Diffuse pain (Y/N) & Duration of emesis (1/2 day intervals) \\
\hline Surgery Performed (yes/no) & Duration of constipation (1/2 day intervals) \\
\hline SBI at surgery (SSBI: yes/no) & Duration of obstipation (1/2 day intervals) \\
\hline Any Emesis (yes/no) & Any Constipation or Obstipation (yes/no) \\
\hline History of Prior Abdominal Surgery (yes/no) & \\
\hline
\end{tabular}

Table 2. Clinical Factors Reviewed from Patient Records

Clinical Variables

History of Abdominal Surgery (\#yes/\#no)

Any Emesis (\#yes/\#no)

Constipation or Obstipation (\#yes/\#no)

Obstructive (Emesis and constipation or obstipation)

Symptoms (\#yes/\#no)

Focal Abdominal Pain (\#yes/\#no)

Radiographic Variables

Free Intraabdominal Fluid (\#yes/\#no)

Fat Stranding (\#yes/\#no)

Bowel Dilation Outside Intussusception (\#yes/\#no)

Lymphadenopathy (\#yes/\#no)

Table 3. Univariate Analysis of Categorical Clinical and Radiographic Variables

\begin{tabular}{ccc|} 
SSBI & No SSBI & p-value \\
\hline $4 / 15$ & $7 / 110$ & 0.025 \\
\hline $9 / 57$ & $2 / 71$ & 0.017 \\
\hline $3 / 14$ & $5 / 103$ & 0.042 \\
\hline $2 / 7$ & $6 / 110$ & 0.044 \\
& & \\
\hline $8 / 46$ & $1 / 68$ & 0.005 \\
\hline & & \\
SSBI & No SSBI & p-value \\
\hline $3 / 14$ & $7 / 109$ & 0.090 \\
\hline $3 / 4$ & $7 / 119$ & $<0.001$ \\
\hline $4 / 8$ & $6 / 114$ & $<0.001$ \\
\hline $4 / 12$ & $4 / 90$ & 0.003 \\
\hline
\end{tabular}


Clinical Variables

Duration of Pain (days)

Duration of Emesis (days)

Duration of Constipation (days)

Radiographic Variables

Length of Intussusception (cm)

Outside Diameter of Intussusception (cm)

Greatest Bowel Wall Thickness of Intussusception (mm)

Table 4. Univariate Analysis of Continuous Clinical and Radiographic Variables
SSBI No SSBI p-value

$\begin{array}{lll}14.1 & 5.0 & 0.025\end{array}$

$13.1 \quad 1.6$

$<0.001$

1.0

1.6
0.079

\section{SSBI}

No SSBI

p-value

9.0

2.9

2.7

$<0.001$

$1.8<0.001$

$4.3<0.001$

\begin{tabular}{|lcc|} 
Multivariate Logistic Regression & Odds Ratio & p-value \\
\hline History of Abdominal Surgery & 7.18 & 0.038 \\
\hline Presence of Focal Abdominal Pain & 22.11 & $<0.001$ \\
\hline Length of Intussusception (cm) & 10.59 & $<0.001$ \\
\hline
\end{tabular}

Table 5. Multivariate Logistic Regression Significant Variables 